\title{
A INFLUÊNCIA DOS PROFISSIONAIS DE SAÚDE SOBRE A PRÁTICA DE ATIVIDADE FÍSICA NO LAZER EM GESTANTES
}

\author{
Simone Cristina Scarpa Romero \\ Universidade de São Paulo, São Paulo, São Paulo, Brasil \\ Carolina Harumi Kurashima \\ Universidade de São Paulo, São Paulo, São Paulo, Brasil \\ Alexandre Romero \\ Universidade de São Paulo, São Paulo, São Paulo, Brasil \\ Monica Yuri Takito \\ Universidade de São Paulo, São Paulo, São Paulo, Brasil
}

\section{Resumo}

O objetivo do presente estudo é analisar a associação entre as recomendações recebidas durante o atendimento pré-natal com a prática de atividade física na gestação. Trata-se de um estudo transversal com 290 puérperas. Apenas 9\% das mulheres atingiam a recomendação ( $\geq 150$ minutos) de atividade física semanal para manutenção da saúde e $18 \%$ praticaram alguma atividade física no lazer (AFL)/exercício físico na gestação. A prática de atividade física anterior à gestação estava associada ao nível de AFL. Adicionalmente, a percepção da presença de recomendação do profissional para a prática de AFL no pré-natal triplicou a chance da mulher ser ativa, ainda que não suficientemente ativa. Ações junto aos profissionais de saúde podem promover modificações no nível de AFL durante a gestação.

Palavras-chave: Gestante; Atividade Física; Recomendação; Profissional de Saúde.

\section{Introdução}

A prática regular de atividade física no lazer é indicada para gestantes devido aos benefícios maternos e fetais que podem atuar em curto ou em longo prazo (IMPACT, 2006). Ademais, a inatividade física durante o período gestacional está associada a desfechos prejudiciais maternos e fetais, como o ganho de peso excessivo, obesidade, diabetes gestacional, pré-eclâmpsia/eclampsia (DEVLIEGER; GUELINCKX; VANSANT, 2008; GUELINCKX et al., 2008), prematuridade e o baixo peso ao nascer (MISRA et al., 1998; TAKITO; BENICIO; NERI, 2009).

Apesar de diretrizes indicando potenciais benefícios para a saúde materno-fetal terem sido publicadas há mais de uma década para nortear a promoção da atividade física (ACOG, 2003) e o exercício físico (WOLFE; DAVIES, 2003), a prática regular é pouco frequente no período gestacional. Como observado por Domingues e Barros (2007) em estudo de coorte populacional, em que apenas $4,7 \%$ das mulheres mantiveram-se ativas durante todo 
o período gestacional. Esta redução do nível de atividade física ocorre tanto em mulheres ativas no período pré-gestacional que descontinuam a prática durante a gestação, assim como nas mulheres que eram sedentárias, as quais são pouco estimuladas a adotarem um estilo de vida ativo (DOMINGUES; BARROS, 2007; TAVARES et al., 2009; EVENSON; WEN, 2011).

Há pouca recomendação para a prática de atividade física no lazer durante a gestação por parte dos profissionais de saúde durante o atendimento de pré-natal (CLARKE; GROSS, 2004; HAAKSTAD; VOLDNER; HENRIKSEN, 2009). Ainda que Entin e Munhall (2006) tenham relatado que metade dos obstetras recomendava a realização de exercícios físicos durante o primeiro e o segundo trimestre da gestação, estes sugeriam uma redução no terceiro trimestre e não informavam qual frequência, duração e intensidade deveria ser realizada.

Estudo recente realizado na cidade de São Paulo com 81 obstetras, Romero et al. (2012) observaram que a recomendação para a prática de exercício físico foi maior no segundo trimestre, sendo a prática da caminhada a atividade mais indicada. Destacou-se um aspecto positivo que a maioria dos médicos apontou diferenças de acordo com o nível de atividade física prévio da mulher. A recomendação também foi mais frequente no serviço privado. Todavia, nota-se que a prática de atividade física no lazer é um problema em todos os níveis socioeconômicos.

Ainda que estes estudos tenham identificado a recomendação dos profissionais para a prática de exercício físico, não foi avaliado o efeito sobre o nível de atividade física no lazer durante a gestação. Dentre os diversos fatores associados à prática de atividade física no lazer, destaca-se na gestação a influência das normas subjetivas percebidas pela indicação ou não da prática pelo profissional de saúde que as acompanha para possibilitar melhor compreensão do porque as mulheres no período gestacional não praticam atividade física visto que a inatividade física é prejudicial à saúde materno-infantil (MISRA et al., 1998; TAKITO; BENICIO; NERI, 2009). Logo, admite-se que a identificação de lacunas entre as recomendações recebidas/percebidas para a prática de atividade física de lazer na gestação, de acordo com o conhecimento vigente, possibilitará o delineamento futuro de intervenções adequadas para melhoria da saúde materno-fetal, com vistas à promoção de um estilo de vida ativo. Desta maneira, o objetivo do presente estudo foi analisar a associação entre as recomendações recebidas durante o atendimento pré-natal com a prática de atividade física no período gestacional.

\section{Métodos}

Trata-se de um estudo transversal com caráter descritivo, com uma amostra composta por 290 puérperas. Foram incluídas mulheres de 18 a 45 anos de idade, no período pós-parto (leito hospitalar). Os critérios de exclusão foram gestação múltipla e presença de patologia ou condição adversa que implique repouso ou redução de atividade física.

O estudo foi realizado no município de Santo André, cidade com 676.407 habitantes, dos quais 52\% são mulheres (IBGE, 2011), localizada na Grande São Paulo. A captação das mulheres foi realizada em dois hospitais no município de Santo André, representando $62 \%$ da média de partos/mês. Partindo de um universo de 7.491 nascimentos de gestação única de mulheres residentes no município de 20 a 39 anos (SINASC, 2011), a amostragem simples apresenta poder amostral superior a 95\% admitindo um erro alfa de 5\% para detectar prevalência menor ou igual a $12 \%$ de mulheres suficientemente ativas (ponto de corte 150 minutos por semana). 
Como instrumento de avaliação foi aplicado um questionário retrospectivo no pós-parto imediato para obter informações referentes aos aspectos demográficos, socioeconômicos, biológicos e antropométricos, obstétricos, hábitos de saúde (tabagismo, etilismo e atividade física) e informações relativas ao aconselhamento ou não para a prática de atividade física no lazer pelo profissional de saúde que acompanhou o pré-natal. O estado nutricional prévio a gestação (WHO, 2000) e no momento do parto (ATALAH et al., 1997) foi classificado para verificar a relação do Índice de Massa Corporal (IMC) com a atividade física. Diante dos diferentes domínios que englobam o termo atividade física (BAUMAN et al., 2012) investigamos a atividade física no lazer/exercício físico e a atividade física global para verificar mudanças de comportamento no domínio específico de atuação do profissional de educação física e no nível de atividade física. O padrão geral de atividade física das mulheres, incluindo seus cinco domínios (atividades ocupacionais, de locomoção, domésticas, lazer e sono), também foi avaliado mediante aplicação do questionário adaptado PPAQ, desenvolvido e validado para gestantes americanas por Chasan-Taber et al. (2004) e brasileiras (TRINDADE, 2007), e recentemente traduzido e utilizado em população brasileira (COLEBRUSCO, 2010). Foram calculados os equivalentes metabólicos semanais (METs) para cada domínio. A partir do total de equivalentes metabólicos foi classificado o nível de atividade física segundo os critérios da FAO/WHO/UNU (2001) em sedentária, pouco ativa, moderadamente ativa e vigorosamente ativa. Em seguida, as categorias foram re-agrupadas em ativas (moderadamente ativas e vigorosamente ativas) e ou não (sedentária ou pouco ativa).

As características da amostra foram descritas utilizando frequências, percentuais, médias e desvios-padrão. Para análise inferencial dos fatores associados à atividade física no lazer e ao nível de atividade física global foram construídos modelos de regressão logística binomial hierárquica, considerando-se $\mathrm{p}<0,20$ como critério para incluir as variáveis de ajuste no modelo (BARROS et al., 2012). Para algumas variáveis foi realizada análise de tendência linear adicional para avaliar o efeito dose-resposta. Para as análises de interesse do presente estudo utilizou-se nível de significância de 5\%.

Todas as mulheres incluídas no estudo consentiram formalmente em participar através do consentimento livre e esclarecido, aprovado pelo Comitê de Ética em Pesquisa Científica (CEPEC-FEFISA), sob o número de protocolo 323/11, e do Comitê de Ética em Pesquisa da Secretaria de Saúde de Santo André (CEPSSSA), sob o número do protocolo $013 / 2012$.

\section{Resultados}

Dentre as 311 mulheres entrevistadas, 21 foram excluídas por apresentarem fatores de exclusão como gestação gemelar $(n=02)$, não realização de pré-natal $(n=01)$, interrupção do preenchimento do questionário $(n=01)$ e contraindicações médicas $(n=17)$ para a realização ou manutenção da atividade física.

A média da idade materna foi igual a 27 anos e o desvio padrão (dp) igual a 06, variando de 18 a 41 anos de idade. A média da renda per capita do grupo de mulheres foi igual a $\mathrm{R} \$ 849,6(\mathrm{dp}=660,4)$. Os valores médios do número de consultas realizadas pelas mulheres durante o pré-natal e o tempo de duração referida pelas puérperas foi igual a $09(\mathrm{dp}=3)$ e 22,5 $(\mathrm{dp}=13,2)$ minutos, respectivamente. Nas variáveis obstétricas, observou-se idade gestacional média igual a 38,7 $(\mathrm{dp}=1,5)$ semanas, peso médio do recém-nascido de 3185,0 $(\mathrm{dp}=458,0)$ gramas e comprimento ao nascer médio igual a 48,3 $(\mathrm{dp}=2,4)$ centímetros. 
A Tabela 01 apresenta a distribuição e proporção das variáveis socioeconômicas, biológicas, obstétricas e estilo de vida das gestantes que participaram do presente estudo. Analisado o nível de escolaridade, observou-se que mais da metade das mulheres frequentou o ensino médio. Quando verificado o estado nutricional observou-se que 51,4\% mulheres apresentavam índice de massa corporal pré-gestacional adequado, no entanto no momento do parto, 53,5\% puérperas apresentavam excesso de peso (ajustado pela idade gestacional).

Em relação ao estilo de vida, 5,9\% cessaram o hábito de fumar durante a gestação e $10,3 \%$ descontinuaram o consumo de bebidas alcoólicas na gestação. Apesar de 21,0\% das mulheres relatarem realizar atividade física no lazer anteriormente ao período gestacional e 18,3\% praticarem alguma atividade física no lazer/exercício físico na gestação, apenas $9 \%$ atingiam a recomendação ( $\geq 150$ minutos/semana).

TABELA 01. Distribuição do número e proporção das variáveis biológicas, obstétricas e estilo de vida das puérperas. Santo André, São Paulo, 2012-2013.

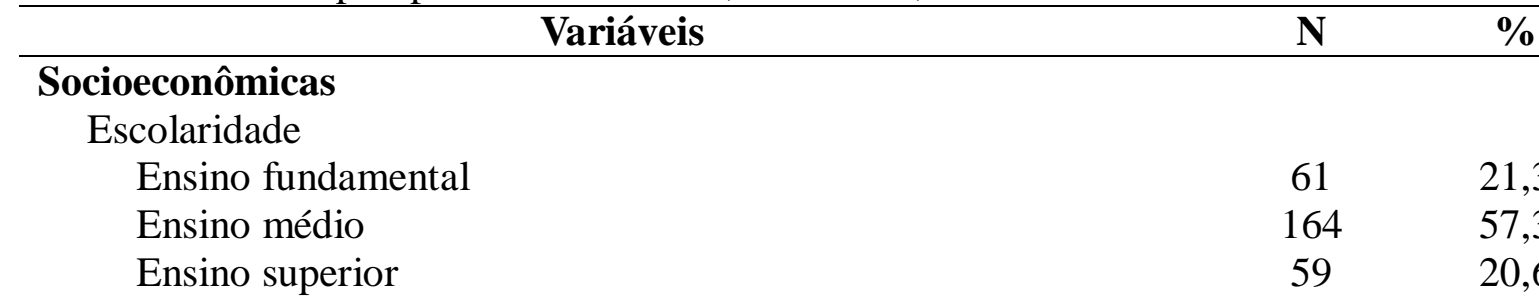

\section{Biológicas}

Estado nutricional pré-gestacional

$\begin{array}{lcc}\text { Abaixo do peso } & 15 & 5,2 \\ \text { Adequado } & 152 & 52,4 \\ \text { Sobrepeso } & 79 & 27,2 \\ \text { Obeso } & 44 & 15,2\end{array}$

\section{Obstétrica}

Tipo de parto

Vaginal 115

39,7

Cesáreo

Momento da primeira consulta de pré-natal

Primeiro trimestre

Segundo trimestre

Terceiro trimestre

\section{Estilo de vida}

Presença de companheiro

Sim

Não

Hábito de fumar

Sim, antes da gravidez

Sim, na gravidez 
Consumo de álcool

Sim, antes da gravidez

30

10,3

Sim, na gravidez

09

3,1

Não

Exerce trabalho remunerado

Sim

Não

Atividade física de lazer pré-gestacional

Sim

Não

Atividade física durante a gestação

Sim

Não

Atinge a recomendação
$\leq 150 \mathrm{~min} / \mathrm{sem}$

O gasto energético dos diferentes domínios da atividade física é apresentado na Tabela 02. Ao observar a atividade física global, constata-se que a atividade física de lazer representa apenas 1,2\% ( $\left.\mathrm{IC}_{95 \%} 0,9-1,6\right)$ do gasto energético diário, enquanto a atividade doméstica corresponde a 54,9\% ( $\mathrm{IC}_{95 \%}$ 52,6-57,1) do gasto energético. Quando comparado o gasto energético diário entre os serviços de saúde, observou-se que as mulheres do serviço público apresentavam maior gasto energético. Entretanto, o percentual de contribuição da atividade doméstica no total da atividade física diferiu significativamente entre os serviços $(\mathrm{p}=0,011)$, representando 57,0\% ( $\mathrm{IC}_{95 \%} 54,5-59,5$ ) nas usuárias do público e 50,9\% ( $\mathrm{IC}_{95 \%}$ 46,4-55,3) nas mulheres do serviço privado (não apresentado em tabela).

TABELA 02. Médias e desvio padrão do gasto energético das puérperas segundo o domínio da atividade física. Santo André, São Paulo, 2012-2013.

\begin{tabular}{lcc}
\hline \multicolumn{1}{c}{ Variáveis } & \multicolumn{2}{c}{ Puérperas $(\mathbf{n = 2 9 0})$} \\
\cline { 2 - 3 } & Média & Desvio Padrão \\
\hline Estilo de vida & & \\
Sono (horas) & 7,84 & $\pm 2,39$ \\
Atividade física global (MET-h/sem) & 31,7 & $\pm 14,4$ \\
Doméstica & 18,0 & $\pm 11,1$ \\
Lazer & 0,4 & $\pm 1,0$ \\
Ocupacional & 7,1 & $\pm 7,9$ \\
Locomoção & 10,9 & $\pm 8,3$ \\
Sedentária & 3,9 & $\pm 2,7$ \\
\hline
\end{tabular}


Segundo a classificação da atividade física global maior escolaridade representa redução da chance da mulher se manter ativa fisicamente. Houve também associação do nível de atividade física global com o estado nutricional no momento do parto, hábito de fumar, número de consultas de pré-natal e o tipo de serviço de saúde. Em relação à prática de atividade física no lazer, observou-se associação positiva com escolaridade, realização de atividade física prévia e presença de conversa ou recomendação do profissional de saúde (TABELA 03).

TABELA 03. Variáveis relacionadas ao nível de atividade física global e no lazer, análise bruta. Santo André, São Paulo, 2012-2013.

\begin{tabular}{|c|c|c|c|c|c|c|c|c|c|c|}
\hline \multirow{3}{*}{ Variáveis } & \multicolumn{5}{|c|}{ ATIVIDADE FÍSICA GLOBAL } & \multicolumn{5}{|c|}{ ATIVIDADE FÍSICA NO LAZER } \\
\hline & \multicolumn{2}{|c|}{ Ativa } & \multirow[b]{2}{*}{ OR } & \multirow[b]{2}{*}{$\mathrm{IC}_{95 \%}$} & \multirow[b]{2}{*}{$p$} & \multicolumn{2}{|c|}{ Praticou } & \multirow[b]{2}{*}{ OR } & \multirow[b]{2}{*}{ IC $95 \%$} & \multirow[b]{2}{*}{$p$} \\
\hline & $\mathbf{N}$ & $\%$ & & & & $\mathbf{N}$ & $\%$ & & & \\
\hline
\end{tabular}

\section{Socioeconômicas}

Renda per capita (salários mínimos/ pessoa) 0,209 0,065

\begin{tabular}{|c|c|c|c|c|c|c|c|c|c|c|}
\hline Até 0,5 & 27 & 44 & 1 & & & 07 & 11 & 1 & & \\
\hline $0,6-1$ & 37 & 44 & 1,00 & 0,52 & 1,93 & 13 & 15 & 1,42 & 0,53 & 3,75 \\
\hline Mais de 1 & 42 & 33 & 0,64 & 0,34 & 1,20 & 31 & 24 & 2,54 & 1,05 & 6,15 \\
\hline
\end{tabular}

Presença de companheiro

0,681

$\begin{array}{lccccc}\text { Não } & 13 & 37 & 1 & & \\ \text { Sim } & 104 & 41 & 1,17 & 0,56 & 2,42\end{array}$

$\begin{array}{lll}04 & 11 & 1\end{array}$

$\begin{array}{lllll}49 & 19 & 1,84 & 0,62 & 5,47\end{array}$

Escolaridade (anos)

0,002

0,012

Até $08 \quad 29 \quad 46 \quad 1$

$0,004 * \quad 07 \quad 11 \quad 1$

$0,007 *$

09 a $11 \quad 74 \quad 45 \quad 0,98 \quad 0,54 \quad 1,75$

$\begin{array}{lllll}24 & 15 & 1,38 & 0,56 & 3,39\end{array}$

12 e mais $\quad 12 \quad 20 \quad \mathbf{0 , 2 9} \quad \mathbf{0 , 1 3} \quad \mathbf{0 , 6 6}$

$\begin{array}{lllll}18 & 30 & \mathbf{3 , 4 3} & \mathbf{1 , 3 1} & \mathbf{8 , 9 6}\end{array}$

\section{Características biológicas}

Idade materna (anos)

0,439

0,803

$\begin{array}{llllll}18 \text { a } 24 & 43 & 43 & 1 & & \\ 25 \text { a } 34 & 62 & 40 & 0,88 & 0,53 & 1,47 \\ 35 \text { a } 41 & 10 & 30 & 0,58 & 0,25 & 1,34\end{array}$

$0,240 * * \quad 17 \quad 17 \quad 1$

$\begin{array}{lllll}30 & 19 & 1,17 & 0,61 & 2,26\end{array}$

$\begin{array}{lllll}05 & 15 & 0,87 & 0,30 & 2,58\end{array}$

IMC pré-gravídico $\left(\mathrm{kg} / \mathrm{m}^{2}\right)^{\S}$

0,461

0,215

$\begin{array}{llllll}\text { Baixo } & 08 & 53 & 1,62 & 0,56 & 4,68\end{array}$

Adequado

$\begin{array}{lll}63 & 41 & 1\end{array}$

$\begin{array}{llllll}\text { Excessivo } & 46 & 37 & 0,84 & 0,52 & 1,37\end{array}$

$$
0,461
$$

$\begin{array}{lllll}04 & 27 & 1,36 & 0,41 & 4,57\end{array}$

$\begin{array}{lll}32 & 21 & 1\end{array}$

$\mathrm{MC}$ parto $\left(\mathrm{kg} / \mathrm{m}^{2}\right)$

$\begin{array}{lllll}17 & 14 & 0,60 & 0,32 & 1,15\end{array}$

$\begin{array}{llllll}\text { Baixo } & 17 & 61 & 1,90 & 0,81 & 4,44\end{array}$

0,015

$\begin{array}{lllllll}\text { Adequado } & 48 & 45 & 1 & \end{array}$

$\begin{array}{lllll}06 & 21 & 1,05 & 0,38 & 2,91\end{array}$

$\begin{array}{lll}22 & 21 & 1\end{array}$

$\begin{array}{llllll}\text { Excessivo } & 52 & 34 & 0,62 & 0,37 & 1,03\end{array}$

$\begin{array}{lllll}25 & 16 & 0,74 & 0,39 & 1,40\end{array}$




\section{Comportamento materno}

Fumo

Não $\begin{array}{lllll}92 & 37 & \mathbf{0 , 4 5} & \mathbf{0 , 2 4} & \mathbf{0 , 8 7}\end{array}$

Sim $\begin{array}{lll}25 & 57 & 1\end{array}$

Bebida

Não

Sim

Trabalho

Não

Sim

Atividade física antes da gestação

Não

$\begin{array}{lll}90 & 39 & 1\end{array}$

Sim

$\begin{array}{lllll}27 & 44 & 1,23 & 0,69 & 2,17\end{array}$

\section{Cuidados de pré-natal}

Consultas (n)

$\begin{array}{llll}\text { Até } 05 & 16 & 62 & 1\end{array}$

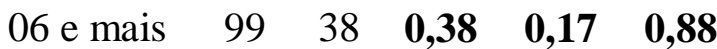

Duração de cada consulta (minutos)

$\begin{array}{llllll}\text { Até } 20 & 84 & 44 & 1,17 & 0,57 & 2,40\end{array}$

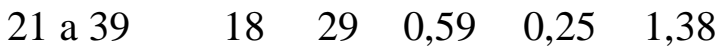

40 e mais $\quad 15 \quad 41 \quad 1$

Atendimento dos profissionais

Diferentes $\quad \begin{array}{lll}20 & 32 & 1\end{array}$

$\begin{array}{llllll}\text { Mesmo } & 97 & 43 & 1,60 & 0,89 & 2,90\end{array}$

Profissional de saúde conversou sobre atividade física no lazer

$\begin{array}{lllcll}\text { Não } & 80 & 43 & 1 & & \\ \text { Sim } & 37 & 35 & 0,70 & 0,43 & 1,14\end{array}$

Profissional de saúde recomendou atividade física no lazer

$\begin{array}{lllcll}\text { Não } & 98 & 44 & 1 & & \\ \text { Sim } & 19 & 28 & \mathbf{0 , 4 9} & \mathbf{0 , 2 7} & \mathbf{0 , 8 9}\end{array}$

Serviço de saúde

$\begin{array}{llllll}\text { Público } & 98 & 52 & \mathbf{4 , 5 4} & \mathbf{2 , 5 6} & \mathbf{8 , 0 7}\end{array}$

0,574

$$
\begin{array}{lll}
25 & 19 & 1
\end{array}
$$

$\begin{array}{lllll}28 & 18 & 0,94 & 0,52 & 1,70\end{array}$

0,153

0,017

0,39

$\begin{array}{lllll}47 & 19 & 1,50 & 0,60 & 3,75\end{array}$

$\begin{array}{lll}06 & 14 & 1\end{array}$

0,137

0,172

$49 \quad 20 \quad 2,12 \quad 0,72 \quad 6,25$

$\begin{array}{lll}04 & 10 & 1\end{array}$

0,833

0,483

$<0,001$

$\begin{array}{lll}25 & 11 & 1\end{array}$

$28 \quad 46 \quad \mathbf{6 , 9 2} \quad \mathbf{3 , 6 1} \quad \mathbf{1 3 , 3 0}$

0,023

0,346

$\begin{array}{lll}03 & 12 & 1\end{array}$

$\begin{array}{lllll}50 & 19 & 1,82 & 0,53 & 6,29\end{array}$

0,089

0,401

$\begin{array}{lllll}31 & 16 & 1,01 & 0,39 & 2,64\end{array}$

$\begin{array}{lllll}15 & 24 & 1,62 & 0,57 & 4,61\end{array}$

$\begin{array}{lll}06 & 16 & 1\end{array}$

0,118

$\begin{array}{lll}14 & 22 & 1\end{array}$

$\begin{array}{lllll}39 & 17 & 0,73 & 0,37 & 1,44\end{array}$

0,361

0,18 $<0,001$

$\begin{array}{lll}30 & 14 & 1\end{array}$

$\begin{array}{lllll}23 & 34 & \mathbf{3 , 2 7} & \mathbf{1 , 7 4} & \mathbf{6 , 1 6}\end{array}$

$<0,001$

$\begin{array}{lll}20 & 11 & 1\end{array}$

$\begin{array}{lllll}33 & 31 & \mathbf{3 , 7 1} & \mathbf{1 , 9 9} & \mathbf{6 , 8 9}\end{array}$

$<0,001$

0,069

$29 \begin{array}{lllll}29 & 15 & 0,57 & 0,31 & 1,05\end{array}$ 


$\begin{array}{lllllll}\text { Privado } & 19 & 19 & 1 & 24 & 24 & 1\end{array}$

SM - salário mínimo; IMC - índice de massa corpórea.

$p$ - nível de significância da regressão logística binomial univariada

* nível de significância segundo o teste de tendência linear

$\S$ Para a análise das características antropométricas (estado nutricional) as variáveis foram recategorizadas.

$\mathrm{Na}$ análise ajustada, conforme apresentado na Tabela 04, pode-se observar que as mulheres atendidas no serviço público têm 3,5 vezes mais chance de serem ativas, quando comparadas às mulheres usuárias do serviço privado. $O$ fato do profissional de saúde recomendar a prática de atividade física no lazer não apresentou significância estatística com o nível de atividade física global, sendo testadas no modelo múltiplo e mantidas junto às demais variáveis de ajuste.

Ao analisar apenas a atividade física no domínio do lazer, observou-se que a mulher que já praticava atividade física anteriormente ao período gestacional apresentava sete vezes mais chance de manter a prática comparada às mulheres que não praticavam previamente. Outro fator importante observado é que a percepção de recomendação do profissional para a prática de atividade física no lazer durante o pré-natal triplicou a chance da mulher praticar quando comparadas às mulheres que não receberam este tipo de orientação.

TABELA 04. Modelo múltiplo dos fatores associados à prática de atividade física global e no lazer. Santo André, São Paulo, 2012-2013.

\begin{tabular}{|c|c|c|c|c|}
\hline Variáveis & OR & \multicolumn{2}{|c|}{ IC $95 \%$} & $p$ \\
\hline \multicolumn{5}{|l|}{ Atividade física global } \\
\hline Serviço de saúde (público) & 3,88 & 1,91 & 7,89 & $<0,001$ \\
\hline Recomendação do profissional de saúde $(\operatorname{sim})^{\S}$ & 0,58 & 0,30 & 1,12 & 0,104 \\
\hline \multicolumn{5}{|l|}{ Atividade física no lazer } \\
\hline Atividade física antes da gestação (sim) ${ }^{\#}$ & 6,90 & 3,31 & 14,37 & $<0,001$ \\
\hline Recomendação do profissional de saúde (sim) ${ }^{\#}$ & 3,10 & 1,48 & 6,50 & 0,003 \\
\hline
\end{tabular}

Quando questionadas sobre possível conversa relacionada à prática de atividade física no lazer $63,4 \%$ das mulheres relataram que não houve troca de informações com o profissional de saúde durante o atendimento pré-natal. Ao analisar os serviços de saúde, observou-se maior frequência de conversa dos profissionais de saúde sobre a prática de atividade física no lazer no privado quando comparado ao serviço público (55\% e 26,8\% respectivamente; $\left.\chi^{2}=22,4 ; p<0,001\right)$, alimentação $\left(77 \%\right.$ e $61,6 \%$ respectivamente; $\chi^{2}=7,04$; $\mathrm{p}=0,008)$, ganho de peso na gestação $\left(81 \%\right.$ e $58,7 \%$ respectivamente; $\left.\chi^{2}=14,54 ; \mathrm{p}<0,001\right)$ e recomendação para a prática de atividade física no lazer $(33 \%$ e $18,4 \%$ respectivamente; $\left.\chi^{2}=7,76 ; p=0,005\right)$. Não foi identificada diferença na abordagem do profissional em relação ao consumo de álcool e tabagismo $\left(51,6 \%\right.$ e $\left.40 \%, \chi^{2}=3,52 ; \mathrm{p}=0,061\right)$.

Apenas $21,0 \%$ das mulheres praticavam algum tipo de atividade anteriormente ao período gestacional e não foi encontrada diferença significativa em relação à recomendação médica segundo a prática prévia de atividade física no lazer $\left(\chi^{2}=0,056 ; p=0,813\right)$. Entretanto, 
as mulheres previamente ativas apresentavam frequência superior de relato de conversa com o profissional sobre a prática de atividade física quando comparadas as mulheres que não realizavam atividade física anteriormente a gestação $(27 \%, 45 \%$ e $17,4 \%$ respectivamente, $\left.\chi^{2}=4,023 ; \mathrm{p}=0,045\right)$. Dentre as previamente ativas $(\mathrm{n}=61), 45,9 \%$ continuaram com a prática após engravidarem, mas ao analisar os diferentes trimestres gestacionais observou que apenas $37,7 \%$ mantiveram-se ativas durante todo o período gestacional, $5,7 \%$ praticaram apenas no primeiro trimestre, $15,1 \%$ no segundo e $7,5 \%$ no terceiro trimestre. Dentre o grupo de mulheres que não praticavam nenhuma atividade física prévia $(n=204), 12,2 \%$ passaram a praticar durante o período gestacional.

Ao avaliar a prática de atividade física realizada, identificamos que dentre as mulheres que praticaram alguma atividade física no lazer durante a gestação, metade fazia menos de 150 minutos de atividade por semana, não atingindo o valor recomendado (TABELA 05). Adicionalmente, 53,8\% das praticantes consideravam o esforço leve/fraco. Em relação à quantidade de atividade física no lazer realizada pelas mulheres e o serviço de saúde, observou-se que o valor recomendado foi atingido mais frequentemente pelas usuárias do serviço público.

TABELA 05. Distribuição do número e proporção de puérperas, segundo a quantidade de atividade física no lazer semanal e a presença de recomendação pelo profissional de saúde. Santo André, São Paulo, 2012-2013.

\begin{tabular}{|c|c|c|c|c|c|c|c|c|}
\hline \multirow{3}{*}{$\begin{array}{c}\text { Informações relativas } \\
\text { à atividade física no } \\
\text { lazer }\end{array}$} & \multicolumn{6}{|c|}{ Quantidade semanal praticada pelas puérperas } & \multirow{3}{*}{$\chi^{2}$} & \multirow{3}{*}{$p^{*}$} \\
\hline & \multicolumn{2}{|c|}{ Não fez } & \multicolumn{2}{|c|}{$\begin{array}{c}\text { Insuficiente } \\
\text { (<150min) }\end{array}$} & \multicolumn{2}{|c|}{$\begin{array}{l}\text { De acordo com o } \\
\text { recomendado } \\
(\geq 150 \text { minutos })\end{array}$} & & \\
\hline & $\mathbf{N}$ & $\%$ & $\mathbf{N}$ & $\%$ & $\mathbf{N}$ & $\%$ & & \\
\hline \multicolumn{7}{|c|}{ Recebeu recomendação do profissional de saúde } & 18,91 & $<0,001$ \\
\hline Não & 192 & 86,5 & 12 & 5,4 & 18 & 8,1 & & \\
\hline Sim & 45 & 66,2 & 15 & 22,1 & 08 & 11,7 & & \\
\hline Setor de atendimento & & & & & & & 8,107 & 0,017 \\
\hline Público & 161 & 84,7 & 11 & 5,8 & 18 & 9,5 & & \\
\hline Privado & 76 & 76,0 & 16 & 16,0 & 08 & 8,0 & & \\
\hline
\end{tabular}

*teste Qui-quadrado $\left(\chi^{2}\right)$.

\section{Discussão}

A prevalência da prática regular de atividade física de lazer foi baixa, apresentando diminuição ao longo da gestação como observado em estudos nacionais e internacionais (DOMINGUES; BARROS, 2007; HAAKSTAD; VOLDNER; HENRIKSEN, 2009). Adicionalmente, dentre as poucas mulheres que praticaram atividade física no lazer esta foi abaixo da recomendação (ACOG, 2003). Atualmente a atividade física no lazer tem se apresentado como uma problemática global e questionamentos recentes têm apontado dificuldades para promover a modificação do comportamento na população (HALLAL et al., 2012), e durante a gestação (CURRIE et al., 2013; PEARCE et al., 2013). As particularidades deste momento fazem com que a norma subjetiva da mulher - como ela percebe a influência da prática de atividade física no lazer para sua saúde e para a saúde de seu bebê - seja 
altamente influenciada pelo prestador de serviço responsável pelo seu cuidado (DUMITH et al., 2012; MAY et al., 2013). Contraditoriamente, o foco das intervenções tem se mantido sob a responsabilidade individual da mulher e desconsiderado a influência do profissional de saúde. Dessa maneira, como apontado por Pearce et al. (2013), sem o envolvimento destes profissionais, menor será o efeito de intervenções sobre o comportamento da mulher. Logo, o presente estudo apresenta um caráter inédito nesse cenário (BAUMAN et al., 2012), uma vez que, apesar das limitações quanto ao desenho transversal, encontrou associação positiva da conduta do profissional sobre o comportamento da mulher - as mulheres que receberam a recomendação do profissional durante o pré-natal tinham três vezes a chance de realizar atividade física no lazer. Como observado no estudo de May et al. (2013), no qual identificou o aconselhamento de um profissional como um determinante da manutenção ou aumento do exercício físico durante a gestação.

Ainda que Dumith et al. (2012) reforce que esta associação encontrada tem potencial para alterar a norma subjetiva podendo ser um indicativo que o quadro atual de inatividade física pode ser modificado com a intervenção do profissional de saúde. Algumas confusões podem surgir ao avaliarmos separadamente ou em conjunto os diferentes domínios da atividade física. No presente estudo, a análise da atividade física global não se apresentou associada à recomendação do profissional de saúde. A hipótese plausível é que a maior contribuição da atividade física doméstica sobre a composição da atividade física global não é afetada pela recomendação. Em relação aos domínios da atividade física, a diferença entre os tipos de serviço de saúde pode ser justificada pelo desencadeamento do fator socioeconômico, ou seja, as mulheres do serviço privado possuem maior renda familiar, que proporciona facilidades, tais como, a contratação de mão de obra terceirizada para a realização das atividades domésticas, a diminuição de atividade de locomoção pela posse de um automóvel, e atividade ocupacional com menor demanda física. Ainda que a prevalência de todos os domínios seja maior nas mulheres usuárias da rede pública, esta é devido ao elevado gasto energético nas atividades domésticas, ocupacionais e de locomoção. Tais atividades apresentam importante contribuição no gasto energético, mas não tem sido positivamente associadas aos desfechos da gestação (TAKITO; BENICIO; NERI, 2009). A diferenciação do domínio de atividade física nas intervenções é de suma importância visto que os desfechos podem ser distintos e nem sempre benéficos. Assim, ainda que a hipótese inicial tenha sido confirmada sendo identificados comportamentos e percepções distintos segundo a condição socioeconômica, o quadro de inatividade física no lazer é preocupante e merece atenção em todos os níveis de atendimento primário a saúde da mulher.

Através de uma revisão sistemática, Currie et al. (2013) observaram que intervenções que proporcionem a mudança de estilo de vida por meio da prática de atividade física pode favorecer a mulher a se manter ativa durante todo o período gestacional. Pensando em estratégias para a promoção da atividade fisica pelos profissionais da saúde, Estabrooks, Glasgow e Dzewaltowski (2003) sugerem que o aconselhamento/orientação da prática de atividade física no lazer seja realizado por enfermeiros, nutricionistas e/ou educadores, visto que às vezes o tempo de consulta médica se torna uma limitação para essa prática. Atualmente no Programa Nacional de Saúde da Família, há a presença do profissional de educação física que pode facilitar esta interação. Joy, Mottola e Chambliss (2013) relatam que deveria existir incentivo, dos órgãos de saúde pública, para que as mulheres se mantivessem ou se tornassem ativas durante a gestação, assim como ferramentas para auxiliar os profissionais de saúde a promover a prática de atividade física no lazer durante todo o pré-natal. Os autores ainda apontam a falta de informação sobre exercício físico na gestação no curso de graduação e/ou 
na formação da residência médica, a falta de tempo nas consultas de pré-natal para aconselhamento sobre estilo de vida, a insegurança da mulher e seu respectivo médico sobre a prática durante o período gestacional e o baixo nível de atividade física prévia a gestação, como sendo possíveis razões para justificar a baixa recomendação de exercício físico para gestantes pelos médicos.

Os resultados obtidos no presente estudo corroboram com este estudo, uma vez que outro fator que se manteve associado à prática de atividade física no lazer na gestação foi o hábito prévio: as mulheres que realizaram atividade física previamente à gestação possuíam sete vezes mais chance de continuar a prática de atividade física no lazer durante o período gestacional. Entretanto, uma limitação a ser discutida é uma possível causalidade reversa, uma vez que a presença de conversa sobre a atividade física no lazer pelo profissional de saúde estava associada à prática de atividade física prévia, podendo ocorrer um viés de memória distinto nos dois grupos (a mulher praticante pode valorizar mais a prática) ou um viés de informação, onde a introdução do assunto nas conversas de pré-natal tenha sido realizada pela mulher e não necessariamente pelo profissional de saúde.

Além disto, a elevada gama de informações e mudanças que a mulher é submetida no período gestacional, podem também fazer com que a mesma não consiga absorver todo o conteúdo discutido durante as consultas. $\mathrm{O}$ atendimento do serviço de pré-natal envolve diversas ações no sentido de reduzir o risco de problemas na gestação, possibilitar ao feto desenvolvimento intrauterino adequado e evitar à mortalidade perinatal. Desta maneira a inclusão de ações preventivas como a recomendação da atividade física no lazer implica em mais uma atividade a ser desenvolvida. Em comparação a orientação da Organização Mundial de Saúde, que recomenda um mínimo de seis consultas para o adequado suporte a saúde da gestante (CARROLI et al., 2001; RASIA; ALBERNAZ, 2008), a presente amostra apresentou elevado número de consultas de atendimento de pré-natal aumentando a possibilidade de abordagem de assuntos e transmissão de informações preventivas. Apesar da maioria dos obstetras considerarem o exercício físico de intensidade leve benéfico o mesmo não ocorre com intensidades moderadas e vigorosas (EVENSON; POMPEII, 2010). O que explica a baixa efetividade da recomendação do profissional de saúde para que as mulheres atendidas no serviço privado atingissem a intensidade moderada e o volume recomendado, apresentando frequência superior de prática insuficiente (abaixo de 150 minutos por semana). Ainda assim, a intenção para a prática e a modificação ou manutenção do comportamento são influenciadas pela percepção do indivíduo e mesmo que esta seja distinta da realidade faz-se necessário a compreensão desta relação. Dessa maneira, seria pertinente investigar as estratégias utilizadas para a recomendação de atividade física durante a gestação, o grau de importância que é dado ao assunto, à regularidade nas consultas, além da frequência, duração e intensidade com que os profissionais de saúde recomendam a prática de atividade física no lazer. Resultados obtidos por Romero et al. (2012) observaram que a maioria dos profissionais recomenda a intensidade leve para as suas pacientes, apesar das evidências atuais que atividades físicas de intensidade moderada a vigorosa seriam benéficas para as mulheres saudáveis durante 0 período gestacional (ZAVORSKY; LONGO, 2011). Neste sentido, a atuação do profissional de educação física junto aos profissionais de saúde, que atuam no atendimento pré-natal, possibilitaria o desenvolvimento de ações de promoção e avaliação da prática regular de atividade física.

Adicionalmente, a investigação dos fatores que envolvem o comportamento e a intenção da gestante possibilitariam outras intervenções para tornar a prática regular um hábito diário. As intervenções que focam a adoção da atividade física de lazer na população 
em geral têm obtido pouco sucesso, devido à falta de suporte teórico para propor modificações de um comportamento complexo (HALLAL et al., 2012; HEATH et al., 2012). O embasamento teórico em futuras intervenções de modificação do comportamento é necessário para uma ação efetiva. Dentre as proposições, os autores destacam a utilização da Teoria do Comportamento Planificado (AJZEN, 1991; 2011) ou de um modelo integrado (HAGGER; CHATZISARANTIS, 2014) com as gestantes, que visam trabalhar os constructos prévios a intenção para a manutenção ou mudança de um comportamento (atitude, norma subjetiva e controle percebido), visando principalmente o constructo da norma subjetiva. A opinião de pessoas próximas e relevantes influencia a intenção do indivíduo para um determinado comportamento. Ainda que revisão sistemática da literatura recente (PEARCE et al., 2013) aponte que o aconselhamento sozinho não apresente efetividade na modificação do comportamento, Downs e Hausenblas (2003) observaram em gestantes que a norma subjetiva é um importante preditor da intenção para se manter ativa.

A realização de novas intervenções com embasamento teórico, com diferentes estratégias para estimular a promoção da prática de atividade física no lazer/exercício físico durante a gestação (MICHIE et al., 2011), deve incluir todos os profissionais de saúde envolvidos para a efetiva mudança de comportamento. Tal proposta deverá ter reforços, ou seja, ser aplicada no início da gestação e ser oferecida em outros momentos durante todo o pré-natal e também após o nascimento, fazendo com que a mulher adote este hábito saudável para a sua vida.

Observa-se a existência de algumas lacunas entre a prática de atividade física no lazer pelas gestantes e a recomendação dos profissionais de saúde, levando as mulheres a diminuírem e/ou pararem a prática. Compreendendo que neste momento específico, a influência do profissional de saúde pode ser um fator determinante, faz-se necessário a elaboração de futuros estudos que incluam a modificação da norma subjetiva com modificações de conduta dos profissionais de saúde durante o atendimento pré-natal minimizando redução da prática de atividade física no lazer. Diante do número de ações a serem realizadas, a elaboração de uma intervenção em prol da adoção de um estilo de vida ativo deve incluir os diversos profissionais envolvidos no atendimento pré-natal, devendo ser integrado desde a anamnese ao acompanhamento das consultas de pré-natal. Entende-se que a capacitação do profissional de saúde sobre a importância da prática regular de atividade física na gestação e como realizar o aconselhamento, seja fundamental para que ocorram recomendações eficientes na promoção da atividade física no lazer entre gestantes tanto no serviço público quanto privado. Sugere-se para futuros estudos, avaliar o impacto de intervenções, para capacitação dos profissionais de saúde, na recomendação da atividade física no lazer para gestantes. Adicionalmente, a proposição de um instrumento que auxilie a equipe de profissionais a promoção da saúde por meio da prática da atividade física, de maneira uniforme, deve ser planejada como uma estratégia para aumentar e/ou manter o nível de atividade física durante a gestação, promovendo a saúde e a qualidade de vida para o binômio mãe-feto.

\section{Considerações finais}

A percepção da presença de recomendação do profissional para a prática de atividade física no lazer durante o pré-natal aumentou a chance de a mulher realizar atividade física no lazer, ainda que não de maneira suficiente. Entende-se que ações junto aos profissionais de saúde podem promover modificações no estilo de vida ativo durante a gestação. Adicionado 
ao fato da prática de atividade física anterior à gestação estar associada ao nível de atividade física no lazer reforçam a importância da promoção destas práticas para mulheres em idade fértil.

Dessa forma, acredita-se que os resultados do presente trabalho possam auxiliar na proposição de ações junto aos profissionais de saúde que atuam no atendimento primário de saúde da mulher e no pré-natal e educadores físicos que atuam junto aos núcleos de apoio ao atendimento à saúde da população feminina para promoção de um estilo de vida ativo.

\title{
THE INFLUENCE OF HEALTH PROFESSIONALS ON THE PRACTICE OF PHYSICAL ACTIVITY IN SPORTS IN PREGNANT WOMEN
}

\begin{abstract}
The aim of this study is to analyze the association between the recommendations received during the prenatal care with the practice of physical activity during pregnancy. This is a cross-sectional study of 290 postpartum women. Only 9\% of women reached the recommendation ( $\geq 150$ minutes) of weekly physical activity for health maintenance and $18 \%$ practiced some physical activity in leisure (LPA) / exercise during pregnancy. The practice of previous physical activity before pregnancy was associated with LPA level. In addition, the perception of professional recommendation of presence to the practice of LPA in the prenatal tripled the chance of women being active, although not sufficiently active. Actions with health professionals can promote changes in LPA level during pregnancy.
\end{abstract}

Keywords: Pregnant Women; Physical Activity, Recommendation, Professional Health.

\section{LA INFLUENCIA DE LOS PROFESIONALES DE LA SALUD EN LA PRÁCTICA DE LA ACTIVIDAD FÍSICA EN DEPORTES EN MUJERES EMBARAZADAS}

\begin{abstract}
Resumen
El objetivo es analizar la asociación entre las recomendaciones durante la atención prenatal y la práctica de la actividad física durante el embarazo. Este estudio transversal con 290 mujeres en el posparto. Sólo $9 \%$ de las mujeres llegó a la recomendación ( $\geq 150$ minutos) de actividad física semanal para mantener la salud y el $18 \%$ practico alguna actividad física en el tiempo libre (LPA)/ejercicio durante el embarazo. La práctica de actividad física previa antes del embarazo se asoció con la LPA. Además, la percepción de la recomendación profesional para LPA en el prenatal se triplicó la probabilidad de que las mujeres ser activa, aunque no suficientemente activa. Acciones con profesionales de la salud pueden promover cambios en el nivel LPA durante el embarazo.
\end{abstract}

Palabras clave: Mujeres Embarazadas; Actividad Física; Recomendación; Personal de Salud.

\section{Referências}

AJZEN, I. The theory of planned behavior. Organizational Behavior and Human Decision Processes, v. 50, n. 02, p, 33, 1991.

AJZEN I. The theory of planned behaviour: reactions and reflections. Psychol Health, v. 26, n. 09, p. 1113-1127, 2011. 
AMERICAN COLLEGE OF OBSTETRICIANS AND GYNECOLOGY [ACOG]. Exercise during pregnancy and the postpartum period. Clin Obstet Gynecol, v. 46, n. 02, p. 496-499, 2003.

ATAlAH, S. E.; CASTILlO, C. L.; CASTRO, R. S. Propuesta de um nuevo estandar de evaluacion nutricional em embarazadas. Rev Med Chile, v. 125, n. 12, p. 1429-1436, 1997.

BARROS, M. V. G de et al. Regressão logística e de Poisson. In: Análise de dados em saúde. Londrina, PR: Midiograf, 2012. p. 215-237.

BAUMAN, A. E. et al. Correlates of physical activity: why are some people physically active and others not? Lancet, v. 380, n. 9839, p. 258-271, 2012.

CARROLI, G. et al. WHO Systematic review of randomised controlled trials of routine antenatal care. Lancet, v. 357, n. 9268, p. 1565-1570, 2001.

CHASAN-TABER, L. et al. Development and validation of a pregnancy physical activity questionnaire. Med Sci Sports Exerc, v. 36, n. 10, p. 1750-1760, 2004.

CLARKE, P. E.; GROSS, H. Women's behaviour, beliefs and information sources about physical exercise in pregnancy. Midwifery, v. 20, n. 2, p. 133-141, 2004.

COLEBRUSCO, L. D. O. Fatores determinantes da variação de peso no período pósparto [dissertação]. São Paulo: Programa Nutrição em Saúde Pública, Faculdade de Saúde Pública, Universidade de São Paulo; 2010.

CURRIE, S. et al. Reducing the decline in physical activity during pregnancy: a systematic review of behaviour change interventions. PLoS One, v. 8, n. 6, p. e66385, 2013.

DEVLIEGER, R.; GUELINCKX, I.; VANSANT, G. The impact of obesity on female reproductive function. Obes Rev, v. 9, n. 2, p. 181-182, 2008.

DOMINGUES, M. R.; BARROS, A. J. D. Leisure-time physical activity during pregnancy in the 2004 Pelotas Birth Cohort Study. Rev Saude Publica, v.41, n. 2, p. 173-180, 2007.

DOWNS, D. S.; HAUSENBLAS, H. A. Exercising for two: examining pregnant women's second trimester exercise intention and behavior using the framework of the theory of planned behavior. Womens Health Issues, v. 13, n. 6, p. 222-228, 2003.

DUMITH, S. C. et al. Atividade física durante a gestação e associação com indicadores de saúde materno-infantil. Rev Saude Publica, v. 46, n. 2, p. 327-333, 2012.

ENTIN, P.; MUNHALL, K. Recommendations regarding exercise during pregnancy made by private/small group practice obstetricians in the USA. J Sports Sci Med, v. 5, n. 3, p. 449-58, 2006. 
ESTABROOKS, P. A.; GLASGOW, R. E.; DZEWALTOWSKI, D. A. Physical activity promotion through primary care. JAMA, v. 289, n. 22, p. 2913-2916, 2003.

EVENSON, K. R.; WEN, F. Prevalence and correlates of objectively measured physical activity and sedentary behavior among US pregnant women. Prev Med, v. 53, n. 1, p. 39-43, 2011.

FAO. Human energy requirements. Food and nutrition technical report series Report of a Joint FAO/WHO/UNU Expert Consultation Rome, 2001. Disponível em: http://www.fao.org/docrep/007/y5686e/y5686e07.htm. Acessado em: 21 de novembro de 2011

GUELINCKX, I. et al. Maternal obesity: pregnancy complications, gestational weight gain and nutrition. Obes Rev, v. 9, n. 2, p. 140-150, 2008.

HAAKSTAD, L. A. ; VOLDNER, N. ; HENRIKSEN, T. BØ K. Why do pregnant women stop exercising in the third trimester? Acta Obstet Gynecol Scand, v. 88, n. 11, p. 1267$1275,2009$.

HAGGER, M. S.; CHATZISARANTIS, N. L. An integrated behavior change model for physical activity. Exerc Sport Sci Rev, v. 42, n. 2, p. 62-69, 2014.

HALLAL, P. C. et al. Physical activity: more of the same is not enough. Lancet, v. 380, n. 9839, p. 190-191, 2012.

HEATH, G. W. et al. Evidence-based intervention in physical activity: lessons from around the world. Lancet, v. 380, n. 9838, p. 272-281, 2012.

IMPACT of physical activity during pregnancy and postpartum on chronic disease risk. Med Sci Sports Exerc, v. 38, n. 5, p. 989-1006, 2006.

Instituto Brasileiro de Geografia e Estatística [IBGE]. Disponível em: http://www.ibge.gov.br/home/estatistica/populacao/censo2010/indicadores_sociais_municipai s/indicadores sociais municipais tab uf zip.shtm

Acessado em: 21 de novembro de 2011

JOY, E. A.; MOTTOLA, M. F.; CHAMBLISS, H. Integrating exercise is medicine ${ }^{\circledR}$ into the care of pregnant women. Curr Sports Med Rep, v.12, n. 4, p. 245-247, 2013.

MAY, L. E. et al. Exercise during pregnancy: the role of obstetric providers. J Am Osteopath Assoc, v. 113, n. 8, p. 612-619, 2013.

MICHIE, S. et al. A refined taxonomy of behavior change techniques to help people change their physical activity and healthy eating behaviors: The CALORE taxonomy. Psychol Health, v. 26, n. 11, p. 1479-1498, 2011.

MISRA, D. P. et al. Effects of physicalactivity on preterm birth. Am J Epidemiol, v. 147, n. 7, p. 628-635, 1998. 
PEARCE, E. E. et al. Strategies to promote physical activity during pregnancy: a systematic review of intervention evidence. Am J Lifestyle Med, v. 7, n. 1, 2013.

RASIA, I. C. R. B.; ALBERNAZ, E. Atenção pré-natal na cidade de Pelotas, Rio Grande do Sul, Brasil. Rev Bras Saude Matern Infant, v. 8, n. 4, p. 401-410, 2008.

ROMERO, S. C. S. et al. Condutas de obstetras acerca do exercício físico na gestação. Revista Corpoconsciência, v. 16, n. 2, p. 46-60, 2012.

SINASC - Sistema de Informações de Nascidos Vivos. Disponível em: http://www.saude.sp.gov.br/. Acessado em: 21 de novembro de 2011.

TAKITO, M. Y.; BENÍCIO, A. M. H.; NERI, L. de C. L. Physical activity by pregnant women and outcomes for newborns: a systematic review. Rev Saude Publica, v. 43, n. 6, p. 1059-1069, 2009.

TAVARES, J. de S. et al. Padrão de atividade física entre gestantes atendidas pela estratégia saúde da família de Campina Grande - PB. Rev Bras Epidemiol, v. 12, n.1, p. 10-19, 2009.

TRINDADE, F. S. Avaliação do nível de atividade física durante a gestação [dissertação]. Ceará: Faculdade de Saúde Pública da Universidade Estadual do Ceará; 2007.

WOLFE, L. A.; DAVIES, G. A. Canadian guidelines for exercise in pregnancy. Clin Obstet Gynecol, v. 46, n. 2, p. 488-495, 2003.

WORLD HEALTH ORGANIZATION [WHO]: Obesity: preventing and managing the global epidemic. Report of a WHO consultation. Technical Report Series no. 894, Geneva: WHO; 2000 .

ZAVORSKY, G. S.; LONGO, L. D. Exercise guidelines in pregnancy: new perspectives. Sports Med, v. 41, n. 5, p. 345-360, 2011.

Recebido em: 25/02/2015

Revisado em: 23/04/2015

Aprovado em: 17/08/2015

Endereço para correspondência:

Simone Cristina Scarpa Romero

simone.scarpa@usp.br

Universidade de São Paulo,

Cidade Universitária "Armando Salles Oliveira",

Butantã - São Paulo - SP - CEP 05508-900 